\title{
Reproductive status affects the expression of prolactin receptor mRNA in the brain of female Damaraland mole-rats
}

\section{Cornelia Voigt* and Nigel Bennett}

Department of Zoology and Entomology, University of Pretoria, 0028 Pretoria, South Africa

Cornelia Voigt, Department of Zoology and Entomology, University of Pretoria, 0028 Pretoria, South Africa, cornelia.voigt@gmail.com

Nigel Bennett, Department of Zoology and Entomology, University of Pretoria, 0028 Pretoria, South Africa, ncbennett@zoology.up.ac.za

* corresponding author:

Cornelia Voigt, Department of Zoology and Entomology, University of Pretoria, 0028 Pretoria, South Africa

Phone: +27-12-4204872, Fax: +27-12-3625242

Email: cornelia.voigt@gmail.com

Short title: Breeding status affects prolactin receptor expression

\section{Highlights}

- Plasma prolactin levels similar in breeding and nonbreeding Damaraland mole-rats.

- Reproductive suppression associated with differential neural Prlr gene expression.

- Elevated Prlr levels in choroid plexus and arcuate nucleus of suppressed females. 


\section{Abstract}

The eusocial Damaraland mole-rat (Fukomys damarensis) represents an extreme example of reproductive skew, in that reproduction is completely blocked in female subordinate group members. It is thought that in these animals normal GnRH secretion from the hypothalamus is disrupted. Prolactin, a peptide hormone secreted from the anterior pituitary gland, has been implicated in a wide variety of functions. Well documented in rodents is its role in mediating lactational infertility. Elevated circulating prolactin levels, such as during lactation, are associated with reduced GnRH release into the portal blood and with a reduction in the frequency and amplitude of LH pulses. The present study aimed at investigating whether such a mechanism could act in reproductively suppressed female Damaraland mole-rats. By means of in situ hybridisation we studied the distribution and gene expression of the prolactin receptor $(P r l r)$ in wild-caught female Damaraland mole-rats with different reproductive status. Substantial Prlr expression was found in several brain regions, with highest levels in the choroid plexus and moderate expression in the preoptic and tuberal hypothalamus. While in reproductive and non-reproductive females plasma prolactin levels were very low and not significantly different, quantification of the Prlr hybridisation signal revealed significant differences in relation to reproductive status. Reproductively suppressed females had increased expression of Prlr in the choroid plexus and in the arcuate nucleus (ARC) when compared to reproductive females. This suggests higher local prolactin levels in the brain of suppressed females. Together with previous findings, it could indicate that prolactin inhibits ARC kisspeptin neurons, which then would lead to reduced activation of GnRH neurons in such females.

KEYWORDS: prolactin receptor, social status, in situ hybridization, Fukomys damarensis, prolactin 


\section{Introduction}

In mammals, prolactin receptors have been found in a wide variety of tissues and consequently, numerous biological functions of prolactin have been described (Kelly et al. 1991; Bole-Feysot et al. 1998; Freeman et al. 2000). The neuroendocrine functions of prolactin include the stimulation of maternal behaviour, suppression of fertility, suppression of stress response, regulation of food intake and body mass and activation of hypothalamic dopaminergic neurons to regulate its own secretion (Grattan and Kokay 2008). Well documented in humans and rodents is the suppression of reproduction during lactation, where high circulating levels of prolactin are associated with an inhibition of gonadotropin secretion and leading to anoestrus or amenorrhoea. Similar effects are also observed in hyperprolactinemic non-lactating females (McNeilly 1980, 2001).

In group-living mammals, reproductive suppression, where dominant individuals inhibit the reproductive capabilities of subordinates via behavioural and other interactions, is commonly found (Keller and Reeve 1994). Extremes of high reproductive skew are represented by societies with a single breeding pair and helpers, such as in callitrichid primates (Abbott 1984), mongooses and meerkats (Rasa 1973, Doolan and Macdonald 1997) and African mole-rats (Bennett and Faulkes 2000). The mole-rats of the family Bathyergidae exhibit a wide range of social behaviour and restricted reproduction has been found in several species (Bennett and Faulkes 2000). Highly social Damaraland mole-rats (Fukomys damarensis) represent an extreme example of reproductive skew, in that reproduction is completely blocked in female subordinate group members (Bennett 1994, Bennett et al. 1994). This blockade results from an inhibition of ovulation, which is caused by a disruption in the normal GnRH secretion from the hypothalamus (Molteno et al. 2004). Despite the potential for prolactin in being involved in the mechanism of reproductive suppression in highly social African mole-rats, its possible role remains elusive. 
In rats and mice, elevated circulating levels of prolactin (hyperprolactinemia) are associated with a reduction in the frequency and amplitude of LH pulses and a suppression of GnRH release into the portal blood (Koike et al. 1984, Cohen-Becker et al. 1986, Fox et al. 1987, Grattan et al. 2007). The reduced activity of the GnRH neurons is considered the principal cause of infertility. This suppressive effect of prolactin is most likely mediated indirectly through prolactin-sensitive afferents such as kisspeptin because only a small percentage of GnRH neurons express the prolactin receptor (Prlr, Grattan et al. 2007, Kokay et al. 2011). Prolactin secretion is under the control of dopamine, which is produced in several distinct hypothalamic dopaminergic neuron populations that express prolactin receptor. Increased prolactin levels lead to the activation of the tuberoinfundibular dopaminergic neurons (TIDA) and to increased dopamine synthesis, which after transport to the anterior pituitary binds to type-2 dopamine receptors and inhibits prolactin secretion. During lactation, the state of physiological hyperprolactinemia, this feedback mechanism is not functional and the elevated prolactin level does not activate TIDA neurons (Grattan and Kokay 2008).

Kisspeptin, a product of the Kissl gene, is a strong activator of GnRH neurons and is considered essential for reproduction and the timing of puberty onset in mammals. Kiss Iexpressing neuron populations have been found mainly in two regions of the hypothalamus, the anteroventral periventricular nucleus (AVPV) and in the arcuate nucleus (ARC, Smith et al. 2006). Prlr is expressed in both Kiss 1 neuron populations (Kokay et al. 2011). In lactating rats, when prolactin levels are elevated, there is a pronounced upregulation of Prlr protein and downregulation of Kiss 1 mRNA and protein expression in the ARC (Pi and Grattan 1999a, Yamada et al. 2007, Araujo-Lopes et al. 2014). Moreover, chronic infusion of prolactin in female mice has been found to suppress oestrus cyclicity, GnRH and LH release and to reduce hypothalamic Kiss1 mRNA expression (Sonigo et al. 2012). These data indicate that the prolactin-induced suppression of GnRH release is mediated by inhibition of Kiss 1 neurons. 
In Damaraland mole-rats we have previously shown that reproductive status affects the neuroendocrine phenotype of females with reproductively suppressed females having reduced gene expression of steroid hormone receptors in several preoptic-hypothalamic brain regions, including the AVPV and the ARC. Moreover, such females also exhibit reduced Kiss1 expression in the ARC compared to reproductively active females (Voigt et al. 2014, Voigt and Bennett 2018). In another highly social African mole-rat, the naked mole-rat (Heterocephalus glaber), transcriptome profiling revealed reduced expression of genes involved in dopamine metabolism in reproductively suppressed subordinate females, which remain in a pre-pubertal anovulatory state (Mulugeta et al. 2017). The present study in Damaraland mole-rats aimed at investigating whether prolactin could play a role in the mechanism of reproductive suppression of subordinate females in this species. We measured circulating prolactin levels and we used in situ hybridization to determine the distribution and gene expression of Prlr in the forebrain of reproductively active and reproductively suppressed females.

\section{Materials and Methods}

\subsection{Animals}

In the current study, adult female Damaraland mole-rats (Fukomys damarensis) were used. Data were obtained from nine reproductives (breeder) and nine nonreproductives (nonbreeder). The females came from colonies that were captured between April and July 2015. Mole-rats were captured near the village of Black Rock, Northern Cape, South Africa $\left(27^{\circ} 7^{\prime} \mathrm{S}, 2^{\circ} 50^{\prime} \mathrm{E}\right)$ with Hickman live-traps under permission from Northern Cape Nature Conservation authorities. Prior to sacrifice, animals were housed for a maximum period of 12 weeks in captivity under a $12 \mathrm{~L}: 12 \mathrm{D}$ cycle at $25^{\circ}$ in plastic containers $(1.0 \mathrm{~m}$ x $0.5 \mathrm{~m} \times 0.5 \mathrm{~m})$ containing wood shavings and they were fed on sweet potato, gem squash and apples. In each colony, the reproductive status was determined for all adults. Breeding females (syn. queens) 
could be readily distinguished from non-reproductive females by the presence of a perforate vagina and prominent teats. None of the females were pregnant or lactating at the time of sacrifice. All animals were kept in their original colonies until sacrifice. At the time of brain collection, body mass of all animals was recorded to the nearest gram. All experimental procedures were approved by the University of Pretoria Animal Ethics Committee (EC00312).

\subsection{Blood sampling and hormone assay}

Upon decapitation of the animal, trunk blood was collected into heparin-coated collection tubes. Blood samples were centrifuged, the plasma collected and frozen at $-40^{\circ} \mathrm{C}$ until hormone assays were conducted. Plasma samples were assayed for prolactin using the commercially available enzyme-linked immunosorbent assay (Elabscience $\odot$ Guinea pig prolactin ELISA kit, Catalogue No: E-EL-GP0358) according to the manufacturer's instructions. The prolactin assay had previously been validated for $F$. damarensis (Bennett et al. 2018). The sensitivity of the assay was $0.1 \mathrm{ng} / \mathrm{ml}$, the detection range $0.16-10 \mathrm{ng} / \mathrm{ml}$, and coefficient of variation for repeatability was $<10 \%$.

\subsection{Brain histology}

Mole-rats were killed by decapitation using a small mammal commercially available guillotine, brains were dissected out of the skull, immediately frozen on dry ice and stored at $80^{\circ} \mathrm{C}$ until used. Before sectioning, brain mass was recorded to the nearest milligram. Frozen brains were cut on a cryostat into $20 \mu \mathrm{m}$ coronal sections. The plane of the sections was adjusted to match as closely as possible the plane of the rat brain atlas (Paxinos and Watson 2007). Sections were mounted onto Superfrost Plus slides (Menzel-Gläser, Braunschweig, Germany). In situ hybridization was carried out on adjacent series of sections for the localization of prolactin receptor (Prlr) mRNA. 


\subsection{Cloning of cDNA probes}

Based on sequence information available from Damaraland mole-rat, PCR was used to amplify a fragment of the Prlr gene. Total RNA was extracted from the mole-rat hypothalamus by using the RNeasy Mini Kit (Qiagen GmbH, Hilden, Germany). The synthesis of first-strand cDNA was done with SUPERSCRIPT III Reverse Transcriptase (Invitrogen, Karlsruhe, Germany) and random primers. The resulting RNA-DNA hybrids were subsequently used in PCR to generate pieces of the appropriate gene. The forward primer was 5'-CCAGGAACCAACGGAGGACT-3' and the reverse primer was 5'AGCCACTGCCCAGACCATAA-3'. PCR was carried out for 40 cycles by using the following parameters: $94^{\circ} \mathrm{C}$ for 1 minute, $55^{\circ} \mathrm{C}$ for 45 seconds, $72^{\circ} \mathrm{C}$ for 1 minute. The amplified fragment was purified and cloned into the pCRII TOPO vector using the TOPO-TA cloning kit (Invitrogen, Karlsruhe, Germany). Resultant clones were sequenced to verify the authenticity and fidelity of the amplification. The cloned Prlr sequence [GenBank: MF944110] is 624bp in length and shows $78 \%$ homology with mouse prolactin receptor [GenBank: NM_011169.5]. Different isoforms of the prolactin receptor have been described in several species including rat and mouse, which differ mainly in the composition of the cytoplasmic domain (Bole-Feysot et al. 1998). Our Prlr sequence covers the extracellular and transmembrane domains (exons 4-8), which are common to short and long forms. Therefore, both isoforms are detected with our probe.

\subsection{In situ hybridization}

The expression of prolactin receptor in brain sections was detected with antisense RNA probes labeled with ${ }^{35}$ S-CTP as described before (Voigt et al. 2014). Labeling of the probes with ${ }^{35} \mathrm{~S}-\mathrm{CTP}$ (1250 Ci/mmol; Perkin Elmer, Rodgau, Germany) was performed using the Riboprobe System (Promega). Our in situ hybridization procedure followed a previously published protocol (Whitfield et al. 1990) with modifications as described elsewhere (Gahr 
and Metzdorf 1997). For signal detection, sections were exposed to autoradiographic film (Kodak Biomax MR, Rochester, NY, USA) for 7 weeks. Brain sections from reproductive and non-reproductive animals were run through the entire procedure at the same time and placed on each autoradiographic film to avoid any possible effect of small differences in procedures on the observed group differences. Control sections processed with the sense probes were obtained from two female mole-rats and were labelled by the same procedure as described above. Autoradiograms from these sections showed no signal. These control data will therefore not be discussed below.

\subsection{Data analysis}

Images from autoradiograms were scanned with an Epson Perfection V750 Pro scanner connected to a PC running the image analysis software Image $\mathrm{J} 1.43 \mathrm{u}(\mathrm{NIH}$, USA; see http://rsb.info.nih.gov/ij/). Before acquisition the system was calibrated by using a calibrated optical density step tablet (T2115CC; Stouffer Industries, Inc., Mishawaka, IN, USA) and a calibration curve was fitted with the Rodbard function of Image $J\left[y=d+(a-d) /\left(1+(x / c)^{\wedge} b\right)\right]$. This calibration was applied to all images and it extended beyond the darkest spot to be measured in the autoradiograms so that the signals that were measured never reached saturation. Regions of interest in each section (defined by the presence of a denser signal density than surrounding areas) were delineated on screen with the computer mouse and their average optical density (OD) was calculated by built-in functions of the software. Background optical density of the film was measured in a rectangular area $\left(1 \mathrm{~mm}^{2}\right)$ in the same image immediately ventral to the brain section of interest. Final OD measurements were obtained by subtracting the film background OD value from the OD value of the region of interest and represent the average measurement from both hemispheres. Brain regions were identified using the atlas of the rat (Paxinos and Watson 2007). Before analysis, sections of all animals were anatomically matched according to the anterior commissure (AC) at the level where it 
reaches its largest extension. For quantification of the mRNA expression levels in the preoptic area (POA), the ARC, the ventrolateral part of the ventromedial nucleus (VMHvl) and the medial nucleus of the amygdala (MeA), adjacent sections along the rostro-caudal axis were measured throughout the extent of the labelling that was visible. Labelling in the POA comprised the AVPV and the medial preoptic nucleus (MPO). For anterior and posterior bed nuclei stria terminalis (aBST, pBST) and for the paraventricular nucleus (PVH), three consecutive sections were measured, respectively. For the choroid plexus (ChP), three consecutive sections at the level of the anterior commissure were measured.

\subsection{Statistical analysis}

Statistical analyses were carried out using JMP software. Data are presented as means \pm SEM. Prlr gene expression was analysed by using a REML-model with reproductive status (breeder vs. non-breeder) and brain region (POA, VMHvl, PVH, ARC, BST, MeA, ChP) as fixed factors and animal ID as random factor. Post hoc analyses were performed with the 'test slices' comparison in JMP. All tests were two-tailed and the significance level was fixed at $\mathrm{p}<0.05$.

\section{Results}

\subsection{Morphological measurements and plasma prolactin levels}

Females of different status differed significantly in body mass with breeders $(115.8 \pm 3.1 \mathrm{~g})$ being heavier than nonbreeders $(83.3 \pm 4.0 \mathrm{~g}, \mathrm{t}=6.32, \mathrm{df}=16, \mathrm{P}<0.0001)$. No significant group differences were found in brain mass $(\mathrm{t}=1.24, \mathrm{df}=16, \mathrm{P}=0.23)$. Plasma prolactin levels of female breeders and nonbreeders were in most animals below the detection limit of the assay. Detectable values were only found in three breeders (mean: $1.98 \pm 1.71 \mathrm{ng} / \mathrm{ml}$, range: $0.27-5.40$ $\mathrm{ng} / \mathrm{ml}$ ) and three nonbreeders (mean: $0.27 \pm 0.06 \mathrm{ng} / \mathrm{ml}$, range: $0.17-0.37 \mathrm{ng} / \mathrm{ml}$ ). The highest prolactin plasma level found was $5.40 \mathrm{ng} / \mathrm{ml}$, which came from a breeding individual. 


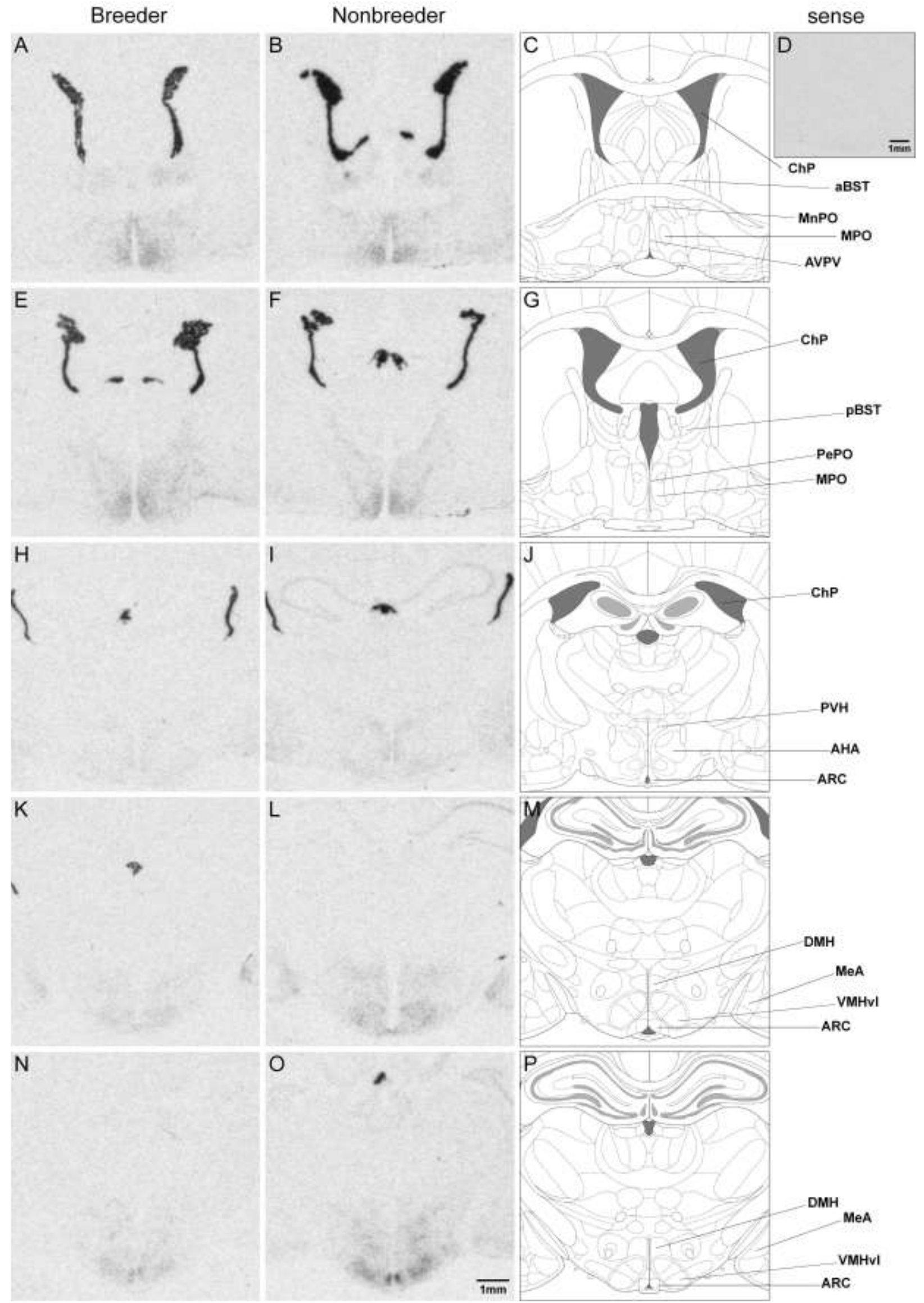

Figure 1. Brightfield photomicrographs of autoradiograms from coronal sections through the mole-rat brain illustrating the distribution of Prlr mRNA visualized by in situ hybridization. Panels illustrate sections from a 
breeding female $(\mathrm{A}, \mathrm{E}, \mathrm{H}, \mathrm{K}, \mathrm{N})$ and a reproductively suppressed female $(\mathrm{B}, \mathrm{F}, \mathrm{I}, \mathrm{L}, \mathrm{O})$ from the level of the preoptic area to the caudal hypothalamus. D: a section hybridized with the sense probe is shown. Abbreviations: AHA, anterior hypothalamic area; ARC, arcuate nucleus; AVPV, anteroventral periventricular nucleus; aBST, bed nucleus of the stria terminalis, anterior part; pBST, bed nucleus of the stria terminalis, posterior part; ChP, choroid plexus; DMH, dorsomedial nucleus; MeA, medial amygdaloid nucleus; MnPO, median preoptic nucleus; MPO, medial preoptic nucleus; PePO, preoptic periventricular nucleus; PVH, paraventricular nucleus; VMHvl, ventromedial hypothalamic nucleus, ventrolateral part.

\subsection{Distribution of prolactin receptor $m R N A$}

Within the hypothalamus, Prlr expression was most pronounced in the preoptic hypothalamus comprising AVPV and the MPO and in the tuberal hypothalamus comprising the ARC and the VMHvl. In other hypothalamic areas weak signals were found in the median preoptic nucleus (MnPO), the preoptic periventricular nucleus (PePO), the anterior hypothalamic area (AHA), the PVH and the dorsomedial nucleus (DMH). Outside the hypothalamus, very strong labelling of Prlr was visible in the ChP. Weaker signals occurred in the anterior and posterior part of the BST, the lateral septum and in the MeA (Fig. 1).

\subsection{Gene expression related to reproductive status}

Measurement of the intensity of the Prlr hybridisation signal in the six different brain areas revealed significant group differences (Fig. 2). The analysis of the average optical density revealed no overall effect of status $\left(\mathrm{F}_{1,16}=4.39, \mathrm{p}=0.053\right)$, but a significant effect of the brain region $\left(\mathrm{F}_{6,96}=398.07, \mathrm{p}<0.001\right)$ and a significant interaction between status and region $\left(\mathrm{F}_{6,96}=3.53, \mathrm{p}=0.003\right)$. Reproductively suppressed females had significantly elevated Prlr expression in the $\mathrm{ChP}$ and in the ARC compared to breeding females. No significant group differences were found in other brain regions. 


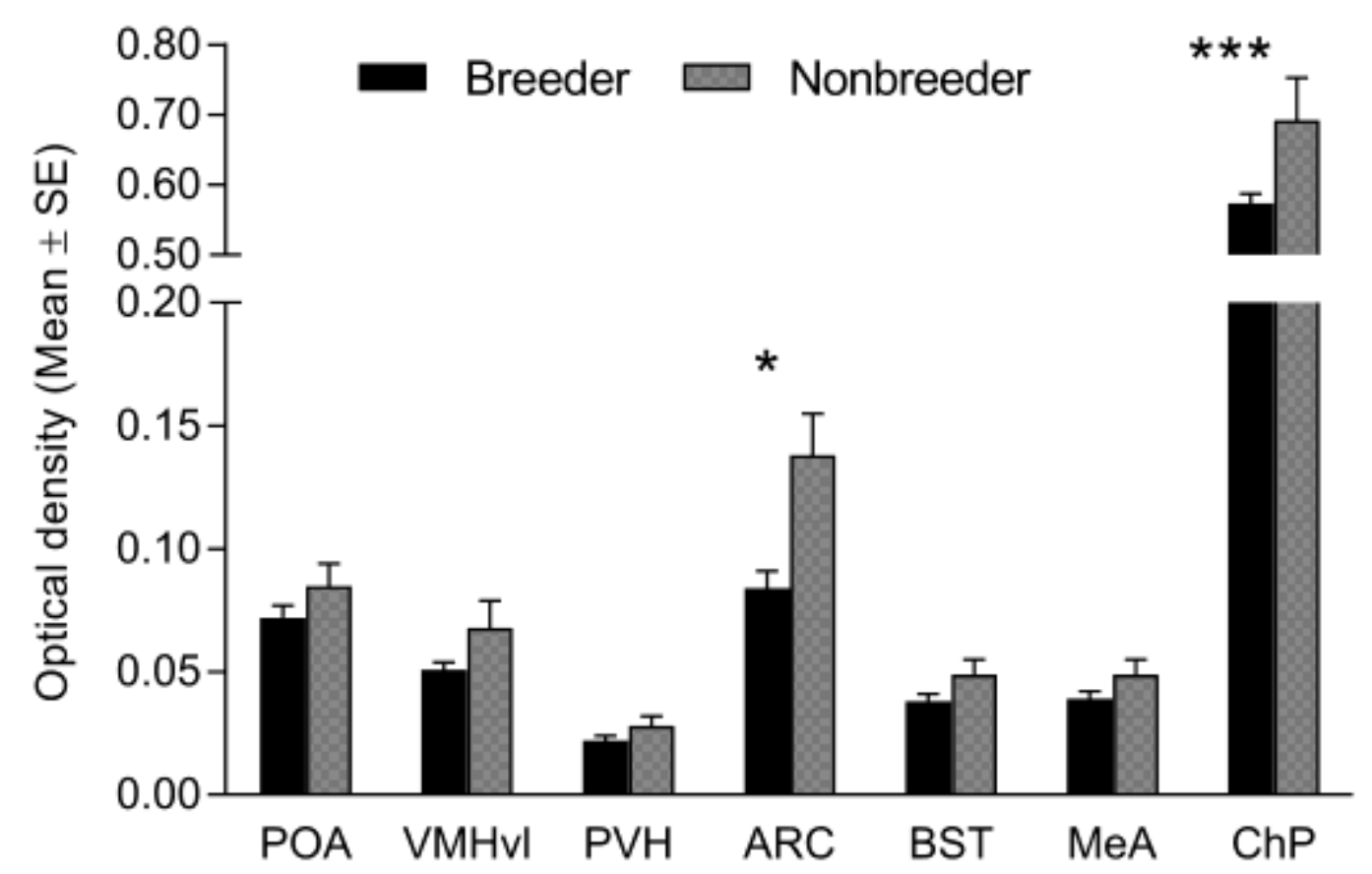

Figure 2. Average optical density of the hybridization signal for Prlr in different brain regions of breeding females and reproductively suppressed females. ${ }^{*} \mathrm{p}<0.05, * * * \mathrm{p}<0.001$.

\section{DISCUSSION}

Here we report for the first time the mRNA expression patterns of Prlr in the brain of female Damaraland mole-rats. In this species, female subordinates, in the presence of the queen, experience strong physiological suppression to the extent of being anovulatory (Bennett 2011). While circulating prolactin levels are very low in both groups of females, by using in situ hybridization, we identify a reproductive status-related difference in Prlr expression in the $\mathrm{ChP}$ and in the $\mathrm{ARC}$, which could play a role in the mechanism of suppression of reproductive behaviour.

\section{Prolactin levels}

Studies in humans and rodents have shown that hyperprolactinemia, a condition of elevated circulating prolactin levels, causes a reduction of $\mathrm{GnRH}$ and $\mathrm{LH}$ secretion and leads to anovulation (Grattan 2015). The physiologically high level of prolactin during lactation is 
maintained because the TIDA neurons in the ARC no longer release dopamine, which under normal conditions inhibits prolactin release (Romano et al. 2013). The hormone data from the present study in Damaraland mole-rats provide no evidence for elevated circulating prolactin levels indicative of hyperprolactinemia in reproductively suppressed females. In both groups of females, circulating prolactin levels were very low or undetectable. It is a limitation to our study that we were not able to assess the oestrous cycle stages using vaginal cytology, therefore, it must be assumed that breeders were in different stages of their oestrous cycle. In rats and mice, circulating prolactin levels peak during proestrous (Butcher et al. 1974; Michael 1976).

It is possible that the hormone level measured in the circulation does not reflect the hormone level in the brain and, thus, reproductively suppressed females could indeed maintain much higher local prolactin levels in the brain extracellular space and in the CSF than those found in the circulation. Prolactin can enter the brain not only from the peripheral circulation, but could reach the hypothalamus and act particularly on the ARC by diffusion from the fenestrated capillaries of the portal blood system in the median eminence. Very high prolactin levels $(4900 \mathrm{ng} / \mathrm{ml})$ are found in the portal plasma of rats while prolactin levels in the pheripheral circulation are low (25 ng/ml; Oliver et al. 1977). Moreover, prolactin can also be produced in the brain (Marano and Ben-Jonathan 2014). Analysis of the brain gene expression of tyrosine hydroxylase, the rate-limiting enzyme in dopamine synthesis, would indicate whether reproductive and non-reproductive females differ in the extent of inhibition by dopamine of prolactin release.

\section{Distribution of Prlr expression}

The distribution of the Prlr mRNA short form and long form has been previously described in the rat and mouse brain (Chiu and Wise 1994, Bakowska and Morrell 1997, Bakowska and Morrell 2003, Brown et al. 2010). The results of the present study in mole-rats, which reports 
very high expression in the $\mathrm{ChP}$ and moderate expression in the preoptic and tuberal hypothalamus, largely agrees with these previous studies. Our probe was designed to detect both the long and short form of the prolactin receptor, therefore we cannot distinguish between their distribution. However, it has been shown that in most areas the distribution of the two isoforms is overlapping (Bakowska and Morrell 2003). One discrepancy to previous studies is the lack of expression in the supraoptic nucleus of mole-rats, which could be explained by the lower sensitiviy of the autoradiographic detection compared to that of silver grains in emulsion coated slides. Interestingly, the distribution of the Prlr gene matches to a large extent the distribution of steroid hormone receptors in the brain of the Damaraland mole-rat. Strong signals of androgen receptor (AR), estrogen receptor $\alpha(\mathrm{ER} \alpha)$ and progesterone receptor (PGR) mRNA expression were previously found in the AVPV, MPO, VMHvl, ARC, BST and MeA (Voigt et al. 2014, Voigt et al. 2016). In rats, oestradiol (E2) and progesterone are involved in the regulation of prolactin receptor gene expression in the brain (Sugiyama et al. 1994; Pi and Voogt 2002; Anderson et al. 2008) and this could also apply to mole-rats.

\section{Breeding status affects Prlr expression}

Quantification of the hybridization signal revealed significant upregulation of the Prlr gene in the $\mathrm{ChP}$ and in the ARC of reproductively suppressed females compared to breeding females despite very low and not significantly different circulating prolactin levels. Previous studies, administering radio-labelled prolactin to rats and mice have shown that high prolactin levels in the peripheral circulation result in increased prolactin uptake at the $\mathrm{ChP}$, measured as the amount of radio-labelled prolactin found in the cerebrospinal fluid (Walsh et al. 1978; Mangurian et al. 1992; Brown et al. 2016). Moreover, during lactation, a physiological condition of chronically high plasma prolactin levels, Prlr mRNA and protein expression at the ChP are significantly increased (Pi and Grattan 1999a; Pi and Grattan 1999b; Augustine et 
al. 2003; Tabata et al. 2012). Also, Prlr protein expression in the rostral and caudal ARC is increased during lactation (Pi and Grattan 1999b). In the light of these data, our findings of increased Prlr expression in the $\mathrm{ChP}$ and ARC of reproductively suppressed females could indicate that local prolactin levels in the brain and CSF are elevated in these females. Alternatively, the low circulating prolactin levels found in these females may reflect the higher uptake of the hormone by Prlr in the brain.

High prolactin levels, such as during lactation, have been shown to suppress Kiss 1 mRNA expression in the ARC and to lead to reduced $\mathrm{LH}$ release in rats and mice (Yamada et al. 2007; Sonigo et al. 2012; Araujo-Lopes et al. 2014; Brown et al. 2014). Prolactin acts directly on ARC Kiss1 neurons as shown by coexpression of Prlr and by induction of pSTAT5 protein expression, a marker for the activation of the long form prolactin receptor in such neurons (Kokay et al. 2011; Araujo-Lopes et al. 2014). Furthermore, treatment of hypothalamic explants containing the median eminence, the ARC and $\mathrm{VMH}$, with prolactin reduces GnRH release and this effect can be reversed by addition of kisspeptin (Sonigo et al. 2012). Kisspeptin is a strong activator of GnRH neurons and is considered essential for reproduction and the timing of puberty onset in mammals (Smith et al. 2006). In Damaraland mole-rats, it is thought that reproductive suppression in subordinate females results from an inhibition of GnRH release from the hypothalamus. In support of this hypothesis, we recently reported reduced Kiss 1 expression and fewer Kiss 1 -expressing cells in the ARC of suppressed compared to breeding females (Voigt and Bennett 2018). Together, these data could indicate that in the former elevated prolactin levels in the brain lead to activation of the Prlr and inhibition of Kissl in ARC kisspeptin neurons, which in turn leads to reduced activation of GnRH neurons and reduced LH release from the pituitary. Future studies involving doublelabel in situ hybridization should confirm colocalization of Prlr and Kiss1 in ARC of Damaraland mole-rats. We previously argued that a long-term negative feedback effect of E2 could be acting on the caudal ARC neurons of reproductively suppressed Damaraland mole- 
rats, leading to decreased levels of Kiss 1 gene expression (Voigt and Bennett 2018) as has been proposed for seasonally anoestrus ewes (Smith et al. 2007). However, the present findings are not contradictory because there is increasing experimental evidence that besides high circulating prolactin levels additional factors must contribute to the suppression of kisspeptin neurons during lactation (Araujo-Lopes et al. 2014; Brown et al. 2014).

Neurons expressing Prlr also exist in the rostral preoptic area, being part of the A15 dopaminergic neurons, which coexpress kisspeptin (Clarkson and Herbison 2011; Brown et al. 2015). In mice, the increased prolactin level during lactation results in suppression of kisspeptin synthesis in these neurons and reduced stimulation of GnRH neurons in this area (Liu et al. 2014). The Kiss 1 neurons in the rostral preoptic area are critical for stimulating the preovulatory GnRH/LH surge. Therefore, one could assume that suppression of these neurons could explain anovulation in subordinate female Damaraland mole-rats. Although we previously found a region-specific downregulation of GnRH mRNA in the rostral preoptic area of reproductively suppressed females (Voigt and Bennett 2017), the lack of differences in Kiss 1 expression in this area (Voigt and Bennett 2018) together with a lack of Prlr upregulation in the present study makes it unlikely that these neuron populations play a major role in a possible prolactin-induced infertility.

In conclusion, we have shown that breeding status affects neural Prlr expression in female Damaraland mole-rats. Although we find no evidence for elevated circulating prolactin levels, the increased Prlr expression at the $\mathrm{ChP}$ and in the ARC suggest higher prolactin levels in the brain of suppressed females. We propose that a mechanism similar to lactation-induced infertility is acting in such females, whereby elevated brain prolactin levels reduce Kiss1 neuron activity in the ARC, leading to reduced activation of GnRH neurons and reduced LH release from the pituitary. Further experimental work is necessary to substantiate this hypothesis. 


\section{COMPETING INTERESTS}

The authors declare that they have no competing interests.

\section{ACKNOWLEDGEMENTS}

This work was supported by a fellowship from the University of Pretoria to CV and funding from the DST-NRF to NB. Permits were obtained from the Northern Cape Department of Nature Conservation. We thank the Family Reynecke for allowing us to work on their farm. We thank Stefanie and Andre Ganswindt for hormone analysis. We are grateful to Manfred Gahr for support.

\section{REFERENCES}

Abbott DH 1984 Behavioral and physiological suppression of fertility in subordinate marmoset monkeys. Am J Primatol 6 169-186.

Anderson GM, Kieser DC, Steyn FJ, Grattan DR 2008 Hypothalamic prolactin receptor messenger ribonucleic acid levels, prolactin signaling, and hyperprolactinemic inhibition of pulsatile luteinizing hormone secretion are dependent on estradiol. Endocrinology 149 $1562-1570$.

Araujo-Lopes R, Crampton JR, Aquino NS, Miranda RM, Kokay IC, Reis AM, Franci CR, Grattan DR, Szawka RE 2014 Prolactin regulates kisspeptin neurons in the arcuate nucleus to suppress LH secretion in female rats. Endocrinology 155 1010-1020.

Augustine RA, Kokay IC, Andrews ZB, Ladyman SR, Grattan DR 2003 Quantitation of prolactin receptor mRNA in the maternal rat brain during pregnancy and lactation. $J \mathrm{Mol}$ Endocrinol 31 221-32.

Bakowska JC, Morrell JI 1997 Atlas of the neurons that express mRNA for the long form of the prolactin receptor in the forebrain of the female rat. J Comp Neurol 386 161-177. 
Bakowska JC, Morrell JI 2003 The distribution of mRNA for the short form of the prolactin receptor in the forebrain of the female rat. Brain Res Mol Brain Res 116 50-58.

Bennett NC 1994 Reproductive suppression in social Cryptomys damarensis colonies: a lifetime of socially-induced sterility in males and females. J Zool (Lond) 234 25-39.

Bennett NC 2011 Teasing apart socially-induced infertility in non-reproductive female Damaraland mole-rats, Fukomys damarensis (Rodentia: Bathyergidae). Integr Zool 6311 320.

Bennett NC, Faulkes CG 2000 African mole-rats. Ecology and eusociality. Cambridge: Cambridge Univ Press.

Bennett NC, Jarvis JUM, Millar RP, Sasano H, Ntshinga KV 1994 Reproductive suppression in eusocial Cryptomys damarensis colonies: socially-induced infertility in females. J Zool (Lond) 233 617-630.

Bennett NC, Ganswindt A, Ganswindt SB, Jarvis JUM, Zoettl M, Faulkes CG 2018 Evidence for contrasting roles for prolactin in eusocial naked mole-rats, Heterocephalus glaber and Damaraland molerats, Fukomys damarensis. Biol. Lett 20180150.

Bole-Feysot C, Goffin V, Edery M, Binart N, Kelly PA 1998 Prolactin (PRL) and its receptor: actions, signal transduction pathways and phenotypes observed in PRL receptor knockout mice. Endocr Rev 19 225-268.

Brown RS, Kokay IC, Herbison AE, Grattan DR 2010 Distribution of prolactin-responsive neurons in the mouse forebrain. J Comp Neurol 518 92-102.

Brown RS, Herbison AE, Grattan DR 2014 Prolactin regulation of kisspeptin neurones in the mouse brain and its role in the lactation-induced suppression of kisspeptin expression. $J$ Neuroendocrinol 26 898-908.

Brown RS, Herbison AE, Grattan DR 2015 Effects of Prolactin and Lactation on A15 Dopamine Neurones in the Rostral Preoptic Area of Female Mice. J Neuroendocrinol 27 708-717. 
Brown RS, Wyatt AK, Herbison RE, Knowles PJ, Ladyman SR, Binart N, Banks WA, Grattan DR 2016 Prolactin transport into mouse brain is independent of prolactin receptor. FASEB J 30 1002-1010.

Butcher RL, Collins WE, Fugo NW 1974 Plasma concentration of LH, FSH, prolactin, progesterone and estradiol-17beta throughout the 4-day estrous cycle of the rat. Endocrinology 94 1704-1708.

Chiu S, Wise PM 1994 Prolactin receptor mRNA localization in the hypothalamus by in situ hybridization. J Neuroendocrinol 6 191-199.

Clarkson J, Herbison AE 2011 Dual phenotype kisspeptin-dopamine neurones of the rostral periventricular area of the third ventricle project to gonadotrophin-releasing hormone neurones. J Neuroendocrinol 23 293-301.

Cohen-Becker IR, Selmanoff M, Wise PM 1986 Hyperprolactinemia alters the frequency and amplitude of pulsatile luteinizing hormone secretion in the ovariectomized rat. Neuroendocrinology 42 328-333.

Doolan SP, Macdonald DW 1997 Band structure and failures of reproductive suppression in a cooperatively breeding carnivore, the slender-tailed meerkat (Suricata suricatta). Behaviour 134 827-848.

Fox SR, Hoefer MT, Bartke A, Smith MS 1987 Suppression of pulsatile LH secretion, pituitary GnRH receptor content and pituitary responsiveness to $\mathrm{GnRH}$ by hyperprolactinemia in the male rat. Neuroendocrinology 46 350-359.

Freeman ME, Kanyicska B, Lerant A, Nagy G 2000 Prolactin: structure, function, and regulation of secretion. Physiol Rev 80 1523-1631.

Gahr M, Metzdorf R 1997 Distribution and dynamics in the expression of androgen and estrogen receptors in vocal control systems of songbirds. Brain Res Bull 44 509-517.

Grattan DR 201560 Years of Neuroendocrinology: The hypothalamo-prolactin axis. $J$ Endocrinol 226 T101-22. 
Grattan DR, Kokay IC 2008 Prolactin: a pleiotropic neuroendocrine hormone. J Neuroendocrinol 20 752-763.

Grattan DR, Jasoni CL, Liu X, Anderson GM, Herbison AE 2007 Prolactin regulation of gonadotropin-releasing hormone neurons to suppress luteinizing hormone secretion in mice. Endocrinology 148 4344-4351.

Keller L, Reeve, KH 1994 Partitioning of reproduction in animal societies. Trends Ecol Evol $998-102$.

Kelly PA, Djiane J, Postel-Vinay MC, Edery M. 1991 The prolactin/growth hormone receptor family. Endocr Rev 12 235-251.

Koike K, Aono T, Miyake A, Tasaka K, Chatani F, Kurachi K 1984 Effect of pituitary transplants on the LH-RH concentrations in the medial basal hypothalamus and hypophyseal portal blood. Brain Res 301 253-258.

Kokay IC, Petersen SL, Grattan DR 2011 Identification of prolactin-sensitive GABA and kisspeptin neurons in regions of the rat hypothalamus involved in the control of fertility. Endocrinology 152 526-535.

Liu X, Brown RS, Herbison AE, Grattan DR 2014 Lactational anovulation in mice results from a selective loss of kisspeptin input to GnRH neurons. Endocrinology 155 193-203.

Mangurian LP, Walsh RJ, Posner BI 1992 Prolactin enhancement of its own uptake at the choroid plexus. Endocrinology 131 698-702.

Marano RJ, Ben-Jonathan N 2014 Minireview: Extrapituitary prolactin: an update on the distribution, regulation, and functions. Mol Endocrinol 28 622-33.

McNeilly AS 1980 Prolactin and the control of gonadotrophin secretion in the female. $J$ Reprod Fertil 58 537-549.

McNeilly AS 2001 Lactational control of reproduction. Reprod Fertil Dev 13 583-590.

Michael SD 1976 Plasma prolactin and progesterone during the estrous cycle in the mouse. Proc Soc Exp Biol Med 153 254-257. 
Molteno AJ, Kallo I, Bennett NC, King JA, Coen CW 2004 A neuroanatomical and neuroendocrinological study into the relationship between social status and GnRH system in cooperatively breeding female Damaraland mole-rats, Cryptomys damarensis. Reproduction 127 13-21.

Mulugeta E, Marion-poll L, Gentie, D, Ganswindt SB, Ganswindt A, Bennett NC, Blackburn EH, Faulkes CG, Heard E. 2017 Molecular insights into the pathways underlying naked mole-rat eusociality. bioRxiv 209932.

Oliver C, Mical RS, Porter JC 1977 Hypothalamic-pituitary vasculature: evidence for retrograde blood flow in the pituitary stalk. Endocrinology 101 598-604.

Paxinos G \& Watson C 2007 The Rat Brain in Stereotaxic Coordinates. Amsterdam, Netherlands: Academic Press.

Pi XJ, Grattan DR 1999a Increased expression of both short and long forms of prolactin receptor mRNA in hypothalamic nuclei of lactating rats. J Mol Endocrinol 23 13-22.

Pi XJ, Grattan DR 1999b Increased prolactin receptor immunoreactivity in the hypothalamus of lactating rats. J Neuroendocrinol 11 693-705.

Pi X, Voogt JL 2002 Sex difference and estrous cycle: expression of prolactin receptor mRNA in rat brain. Brain Res Mol Brain Res 103 130-139.

Rasa A 1973 Intra-familial sexual repression in the dwarf mongoose Helogale parvula. Naturwissenschaften 60 303-304.

Romanò N, Yip SH, Hodson DJ, Guillou A, Parnaudeau S, Kirk S, Tronche F, Bonnefont X, Le Tissier P, Bunn SJ, Grattan DR, Mollard P, Martin AO 2013 Plasticity of hypothalamic dopamine neurons during lactation results in dissociation of electrical activity and release. J Neurosci 33 4424-33.

Smith JT, Clifton DK, Steiner RA. 2006 Regulation of the neuroendocrine reproductive axis by kisspeptin-GPR54 signaling. Reproduction 131 623-630. 
Smith JT, Clay CM, Caraty A, Clarke IJ 2007 KiSS-1 messenger ribonucleic acid expression in the hypothalamus of the ewe is regulated by sex steroids and season. Endocrinology 148 1150-1157.

Sonigo C, Bouilly J, Carré N, Tolle V, Caraty A, Tello J, Simony-Conesa FJ, Millar R, Young J, Binart N 2012 Hyperprolactinemia-induced ovarian acyclicity is reversed by kisspeptin administration. J Clin Invest 122 3791-3795.

Sugiyama T, Minoura H, Kawabe N, Tanaka M, Nakashima K 1994 Preferential expression of long form prolactin receptor mRNA in the rat brain during the oestrous cycle, pregnancy and lactation: hormones involved in its gene expression. $J$ Endocrinol $141325-$ 333.

Tabata H, Kobayashi M, Ikeda JH, Nakao N, Saito TR, Tanaka M 2012 Characterization of multiple first exons in murine prolactin receptor gene and the effect of prolactin on their expression in the choroid plexus. J Mol Endocrinol 48 169-176.

Voigt C, Bennett NC 2017 GnRH mRNA expression in the brain of cooperatively-breeding female Damaraland mole-rats. Reproduction 153 453-460.

Voigt C, Bennett NC 2018 Reproductive status-dependent kisspeptin and RFamide-related peptide (Rfrp) gene expression in female Damaraland mole-rats. J Neuroendocrinol 30 e12571.

Voigt C, Leitner S, Bennett NC 2016 Breeding status affects the expression of androgen and progesterone receptor mRNA in the brain of male Damaraland mole-rats. J Zoology Lond 298 209-216

Voigt C, Gahr M, Leitner S, Lutermann H \& Bennett NC 2014 Breeding status and social environment differentially affect the expression of sex steroid receptor and aromatase mRNA in the brain of female Damaraland mole-rats. Frontiers in Zoology 1138.

Walsh RJ, Posner BI, Kopriwa BM, Brawer JR 1978 Prolactin binding sites in the rat brain. Science 201 1041-1043. 
Whitfield HJ Jr, Brady LS, Smith MA, Mamalaki E, Fox RJ, Herkenham M 1990 Optimization of cRNA probe in situ hybridization methodology for localization of glucocorticoid receptor mRNA in rat brain: a detailed protocol. Cell Mol Neurobiol 10 145-157.

Yamada S, Uenoyama Y, Kinoshita M, Iwata K, Takase K, Matsui H, Adachi S, Inoue K, Maeda KI, Tsukamura H 2007 Inhibition of metastin (kisspeptin-54)-GPR54 signaling in the arcuate nucleus-median eminence region during lactation in rats. Endocrinology 148 2226-2232. 